\title{
Comparison between three oxaliplatin-based regimens with bevacizumab in patients with metastatic colorectal cancer
}

This article was published in the following Dove Press journal:

OncoTargets and Therapy

3 March 2015

Number of times this article has been viewed

\author{
Yoshihito Ohhara ${ }^{1,2}$ \\ Mitsukuni Suenaga' \\ Satoshi Matsusaka' \\ Eiji Shinozaki' \\ Nobuyuki Mizunuma' \\ Toshiharu Yamaguchi ${ }^{3}$ \\ 'Department of Gastroenterology, \\ Cancer Institute Hospital of the \\ Japanese Foundation for Cancer \\ Research, Tokyo, Japan; ${ }^{2}$ Department \\ of Medical Oncology, Hokkaido \\ University Graduate School \\ of Medicine, Sapporo, Hokkaido, Japan; \\ ${ }^{3}$ Department of Gastroenterological \\ Surgery, Cancer Institute Hospital of \\ the Japanese Foundation for Cancer \\ Research, Tokyo, Japan
}

Background: A previous pivotal Phase III study (NO16966) demonstrated the benefit of the addition of bevacizumab (BV) to oxaliplatin-based regimens in metastatic colorectal cancer (MCRC). Our study evaluated the safety and efficacy of three oxaliplatin-based chemotherapy regimens (FOLFOX4 [intravenous twice-bolus and twice-infusional 5-fluorouracil/folinic acid plus oxaliplatin], mFOLFOX6 [intravenous once-bolus and once-infusional 5-fluorouracil/ folinic acid plus oxaliplatin], and XELOX [capecitabine plus oxaliplatin]) plus BV in the firstline treatment of MCRC patients.

Methods: Patients with MCRC who started treatment between June 2007 and September 2010 were evaluated in this retrospective cohort study. We also evaluated early objective tumor response (EOTR) within 12 weeks, which was defined as a relative change of $\geq 30 \%$ in the sum of the longest diameters of target lesions when compared with baseline. The primary study endpoints were progression-free survival (PFS) and response rate.

Results: A total of 185 patients received the following chemotherapy: FOLFOX4 + BV (FF4 arm, $n=85)$, mFOLFOX6 + BV (FF6 arm, n=40), and XELOX + BV (XELOX arm, $n=60)$. The overall response rates were $61.2 \%, 72.5 \%$, and $75.0 \%$ (95\% confidence interval: $50.6 \%-71.8 \%$, $58.0 \%-87.0 \%$, and $63.7 \%-86.3 \%$ ). Median PFS was $18.0,15.5$, and 13.7 months, respectively (log-rank: $P=0.254$; data cut-off: May 2013). Patients with EOTR ( $\mathrm{n}=117)$ had significantly better PFS than those without-EOTR (n=68) (17.5 versus 12.7 months, $P=0.004$ ).

Conclusion: This study suggests that these three BV plus oxaliplatin-based treatments might have comparable benefit in terms of tumor response and PFS. Moreover, EOTR may be a predictive factor for PFS in patients with MCRC.

Keywords: bevacizumab, early tumor objective response, oxaliplatin, colorectal cancer

\section{Introduction}

Colorectal cancer was the third most common malignancy and the second most frequent cause of cancer-related death worldwide in 2008. ${ }^{1}$ Metastases are present in about 25\% to $30 \%$ of patients with colorectal cancer at the time of diagnosis, and will develop in another $25 \%$. Unfortunately, the prognosis is usually limited at this stage of the disease, but systemic chemotherapy can control the disease, alleviate the symptoms related to cancer, and prolong survival. ${ }^{2}$

FOLFOX, a fortnightly schedule of bolus and infusional 5-fluorouracil (5-FU)/folinic acid (FA) plus oxaliplatin, is a widely used regimen for first-line treatment against metastatic colorectal cancer (MCRC). ${ }^{3,4}$ Capecitabine (Xeloda ${ }^{\circledR}$; Chugai-Roche, Tokyo, Japan) is an oral fluoropyrimidine that is equivalent to intravenous 5-FU in tumor tissue and acts by exploiting the increased expression of the enzyme thymidine phosphorylase in tumors. ${ }^{5,6}$ Some clinical studies show that capecitabine can replace bolus 5-FU/FA
Correspondence: Yoshihito Ohhara Department of Gastroenterology, Cancer Institute Hospital of the Japanese Foundation for Cancer Research, 3-8-3I Ariake, Koto-ku, Tokyo I35-8550, Japan Tel +81335200 I I I

Fax +81335700343

Email yoshihito-ohhara@kkr-smc.com 
as first-line therapy for $\mathrm{MCRC}^{7-9}$ and as adjuvant therapy for stage III colon cancer. ${ }^{10}$ Moreover, capecitabine has also been combined with oxaliplatin (XELOX regimen) to produce an effective first-line regimen for MCRC in Phase II studies. ${ }^{1-13}$ A pivotal Phase III study (NO16966) showed that XELOX was noninferior to FOLFOX4 in terms of efficacy as first-line therapy for patients with MCRC. ${ }^{14}$

Bevacizumab (BV) is a recombinant humanized monoclonal antibody to vascular endothelial growth factor (VEGF). The addition of BV to cytotoxic chemotherapy is one of the treatment options for patients with MCRC. The NO16966 study further demonstrated that adding BV to oxaliplatin-based chemotherapy improved progression-free survival (PFS) in the first-line treatment of MCRC. ${ }^{15,16}$ In our retrospective study, adding BV to FOLFOX4 prolonged PFS and overall survival (OS) when compared with FOLFOX4 in Japanese patients with MCRC (PFS, 17.0 versus 9.9 months; hazard ratio $[\mathrm{HR}], 0.58 ; 95 \%$ confidence interval [CI]: $0.42-0.82$; $P=0.002$; OS, 38.8 versus 20.5 months; HR, 0.49; 95\% CI: $0.34-0.71 ; P<0.001) .{ }^{17}$ XELOX was approved for MCRC in September 2009 in Japan. Doi et al described the efficacy and safety of XELOX + BV in Japanese patients with MCRC in a Phase I/II study. ${ }^{18}$ However, there are no data in Japanese patients with MCRC that compare XELOX + BV and FOLFOX (FOLFOX4 or mFOLFOX6) + BV.

Recently, some investigators reported that early tumor shrinkage (ETS) $\geq 20 \%$ in MCRC was associated with prolonged PFS and OS. Modest et al studied patients with KRAS wild-type (WT) MCRC who were treated with a cetuximabcontaining regimen and reported ETS $\geq 20 \%$ at 6 weeks after initiation of chemotherapy in $59 \%$ of patients. ${ }^{19}$ Patients with ETS $\geq 20 \%$ had better overall response rate (ORR) than those with non-ETS ( $82 \%$ versus $19 \% ; P<0.001)$. Moreover, PFS (8.9 months versus 4.7 months, $P<0.001$ ) and OS (31.6 months versus 15.8 months; $P=0.005$ ) were significantly superior in patients with ETS $\geq 20 \%$ when compared with those in non-ETS patients. Giessen et al reported similar results with regard to ETS in patients treated with irinotecan-based combined chemotherapy. ${ }^{20}$ These reports suggest that ETS might be a good predictor of PFS and OS in patients with MCRC. Moreover, Sommeijer et al reported that early objective tumor response (EOTR) between 6 and 12 weeks after initiation chemotherapy was associated with prolonged PFS and OS in patients with MCRC. ${ }^{21}$

The purpose of this retrospective study was to evaluate the safety and efficacy of three oxaliplatin-based chemotherapies (FOLFOX4, mFOLFOX6, and XELOX) plus BV in the firstline treatment of patients with MCRC.

\section{Patients and methods}

\section{Study design}

This was a retrospective study conducted at the Cancer Institute Hospital of the Japanese Foundation for Cancer Research in Tokyo, Japan. Patients with MCRC received FOLFOX4 + BV (FF4 arm) between June 2007 and November 2008, mFOLFOX6 + BV (FF6 arm) between December 2008 and September 2009, and XELOX + BV (XELOX arm) between October 2009 and September 2010. All patients provided written informed consent before undergoing treatment.

\section{Patients}

The eligibility criteria were as follows: 1) histologically proven MCRC; 2 ) age $\leq 75$ years with an Eastern Cooperative Oncology Group (ECOG) performance status (PS) range of $0-2$ at the start of chemotherapy; 3 ) adequate organ function; and 4) no prior systemic chemotherapy for MCRC and no progression within 6 months of adjuvant chemotherapy completion (if received). Some of the exclusion criteria were as follows: brain tumor or brain metastases; urinary protein $\geq 100 \mathrm{mg} / \mathrm{dL}$ within 1 week before treatment; uncontrolled hypertension despite two or more medications; peptic ulcer; or clinically significant cardiovascular disease.

\section{Treatment}

The treatment regimens were: FOLFOX4 + BV $\left(85 \mathrm{mg} / \mathrm{m}^{2}\right.$ intravenous oxaliplatin for 2 hours on day 1 plus $100 \mathrm{mg} / \mathrm{m}^{2}$ l-leucovorin for 2 hours and $400 \mathrm{mg} / \mathrm{m}^{2}$ bolus 5 -FU, followed by a 22-hour infusion of $600 \mathrm{mg} / \mathrm{m}^{2} 5$-FU on days 1 and 2 plus $5 \mathrm{mg} / \mathrm{kg}$ BV for 30-90 minutes on day 1 every 2 weeks); mFOLFOX6 + BV (85 mg/m² intravenous oxaliplatin for 2 hours on day 1 plus $200 \mathrm{mg} / \mathrm{m}^{2} l$-leucovorin for 2 hours and $400 \mathrm{mg} / \mathrm{m}^{2}$ bolus $5-\mathrm{FU}$, followed by a 46 -hour infusion of $2,400 \mathrm{mg} / \mathrm{m}^{2} 5$-FU on day 1 plus $5 \mathrm{mg} / \mathrm{kg} \mathrm{BV}$ for 30-90 minutes on day 1, every 2 weeks), and XELOX + $\mathrm{BV}\left(130 \mathrm{mg} / \mathrm{m}^{2}\right.$ intravenous oxaliplatin for 2 hours on day 1 plus oral capecitabine $1,000 \mathrm{mg} / \mathrm{m}^{2}$ twice daily for 2 weeks from day 1 plus $7.5 \mathrm{mg} / \mathrm{kg}$ BV for 30-90 minutes on day 1 , every 3 weeks).

Treatment was continued until disease progression, intolerable adverse events, or withdrawal of consent. The disease progression was defined as a $\geq 20 \%$ increase in the sum of the longest dimensions of target lesions from baseline, appearance of new lesions, or death, whichever came first. Oxaliplatin was stopped when patients experienced grade 2 or more neurosensory toxicity lasting more than 7 days and was reintroduced if patients recovered from the neurosensory toxicity. When we stopped oxaliplatin, we continued chemotherapy consisting 
of BV plus 5-FU/FA or capecitabine. Antiemetic drugs and dose reduction of the designated drugs were allowed at the discretion of the treating physician.

\section{Efficacy and safety evaluation}

Tumor assessments with computed tomography scan were performed within 4 weeks before the first administration of treatment and were repeated every 8-12 weeks. Objective responses in measurable lesions were evaluated according to the guidelines of the Response Evaluation Criteria in Solid Tumors Committee (RECIST) version 1.0. The objective response was assessed by the physicians.

The first evaluation was performed between 8 and 12 weeks using computed tomography scan, and EOTR was analyzed during this first evaluation. EOTR was defined as a relative change of $\geq 30 \%$ in the sum of the longest diameters of target lesions at the first evaluation when compared with baseline.

Symptomatic toxicities and laboratory data were monitored every cycle at the outpatient department. Toxicities were evaluated according to the Common Toxicity Criteria for Adverse Events (CTCAE), version 4.0. Dose reduction and treatment delays were recommended according to the extent of hematological and non-hematological toxicities.

\section{Statistical analysis}

PFS was defined as the period from the date of the first administration of BV plus oxaliplatin-based chemotherapy to the date of confirmation of tumor progression by imaging, the date of symptomatic deterioration by clinical judgment, or the last date on which the patient was confirmed to be alive without disease progression. If a patient experienced progression on BV plus 5-FU/FA or capecitabine, we counted it as a PFS event. Patients who did not have target lesions were excluded from the waterfall plot analysis. The survival curves were calculated by the Kaplan-Meier method. Predictive factors were established by searching all variables that significantly influenced PFS at a $P$-value of $<0.05$ in the univariate analysis. Multivariate analysis was performed using the Cox proportional hazards regression model. Statistical analyses were performed using the SPSS software package version 18.0 (SPSS Inc., Chicago, IL, USA). $P$-values were two-sided, with $P<0.05$ indicating statistical significance.

\section{Results}

\section{Patient characteristics}

Subjects were patients with MCRC who received BV plus oxaliplatin-based chemotherapy as first-line treatment at the Cancer Institute Hospital of the Japanese Foundation for Cancer Research in Japan. Between January 2007 and November 2008, 85 patients received FOLFOX4 + BV. Between December 2008 and September 2009, 40 patients received mFOLFOX6 + BV. Between October 2009 and September 2010, 60 patients received XELOX + BV. Characteristics of patients of each arm are shown in Table 1. The median age was 60 years (range, 16-74 years) in the FF4 arm, 61 years (range, 43-74 years) in the FF6 arm, and 60 years (range, 38-75 years) in the XELOX arm. Almost all patients in this study had ECOG PS 0 (FF4 arm, 99\%; FF6 arm, 93\%; and XELOX arm, 95\%), and no patients had ECOG PS 2. Primary site resection was performed more commonly in the FF4 (92\%) and FF6 (93\%) arms than in the XELOX arm (72\%). The incidence of liver metastasis in the FF6 arm (30\%) was lower than that in the FF4 (53\%) and XELOX (62\%) arms. However, the incidence of the other metastatic sites was almost similar when comparing the three arms. KRAS status was checked in 111 patients $(60 \%)$; among them, the number of patients with $K R A S$ WT status was 69 patients (62\%), and the number of patients with $K R A S$ mutant-type status was 42 patients (38\%).

\section{Treatment outcome and waterfall plot analysis}

The ORRs (complete response + partial response) of the FF4, FF6, and XELOX arms were $61.1 \%$, $72.5 \%$, and $75 \%$ (95\% CI: 50.6\%-71.8\%, 58.0\%-87.0\%, and 63.7\%-86.3\%), respectively (Table 2). Among them, 117 patients $(63.2 \%)$ achieved early response at the first evaluation (Figure 1). The $\mathrm{R} 0$ resection rate after chemotherapy was $11 \%$ in the FF4 arm, $5 \%$ in the FF6 arm, and $12 \%$ in the XELOX arm. The median relative dose intensities (RDIs) of oxaliplatin were 0.54 (range, 0.14-1) in the FF4 arm, 0.59 (range, 0.23-1) in the FF6 arm, and 0.68 (range, 0.12-1) in the XELOX arm. The median RDIs of 5-FU or capecitabine were 0.71 (range, 0.24-1) in the FF4 arm, 0.72 (range, 0.41-1) in the FF6 arm, and 0.71 (range, 0.51-1) in the XELOX arm. The median combination rates of BV to chemotherapy were 0.94 (range, 0.04-1) in the FF4 arm, 1 (range, 0.07-1) in the FF6 arm, and 0.90 (range, 0.03-1) in the XELOX arm. Secondline chemotherapy was given to more than $70 \%$ patients in each arm.

Median PFS was 18.0 months in the FF4 arm (median follow-up time, 35.0 months), 15.5 months in the FF6 arm (median follow-up time, 30.0 months), and 13.7 months in the XELOX arm (median follow-up time, 24.2 months), respectively (cut-off: May 2013) (Figure 2). There were no 
Table I Patient characteristics

\begin{tabular}{|c|c|c|c|c|c|c|}
\hline & \multicolumn{2}{|c|}{ FOLFOX4 + BV } & \multicolumn{2}{|c|}{ mFOLFOX6 + BV } & \multicolumn{2}{|c|}{ XELOX + BV } \\
\hline & $n=85$ & (\%) & $n=40$ & (\%) & $n=60$ & (\%) \\
\hline \multicolumn{7}{|l|}{ Age (years) } \\
\hline Median (range) & 60 & $(16-74)$ & 61 & $(43-74)$ & 60 & $(38-75)$ \\
\hline \multicolumn{7}{|l|}{ Sex } \\
\hline Male/female & $4 I / 44$ & $(48 / 52)$ & $25 / 15$ & $(63 / 37)$ & $35 / 25$ & $(58 / 42)$ \\
\hline \multicolumn{7}{|c|}{ ECOG performance status } \\
\hline $0 / 1$ & $84 / 1$ & $(99 / 1)$ & $37 / 3$ & $(93 / 7)$ & $57 / 3$ & $(95 / 5)$ \\
\hline \multicolumn{7}{|l|}{ Primary site } \\
\hline Colon/rectum & $62 / 23$ & $(73 / 27)$ & $28 / 12$ & $(70 / 30)$ & $42 / 18$ & $(70 / 30)$ \\
\hline \multicolumn{7}{|c|}{ Stage at first diagnosis } \\
\hline Local regional & 42 & (49) & 29 & (73) & 25 & $(42)$ \\
\hline Metastatic & 43 & $(5 \mathrm{I})$ & 11 & $(27)$ & 35 & $(58)$ \\
\hline \multicolumn{7}{|c|}{ Adjuvant chemotherapy } \\
\hline Yes & 35 & $(4 I)$ & 23 & $(58)$ & 14 & $(23)$ \\
\hline \multicolumn{7}{|c|}{ Resection of primary site } \\
\hline Yes & 78 & $(92)$ & 37 & (93) & 43 & $(72)$ \\
\hline \multicolumn{7}{|l|}{ Metastasis site } \\
\hline Liver & 45 & $(53)$ & 12 & (30) & 37 & $(62)$ \\
\hline Lung & 35 & $(4 I)$ & 17 & (43) & 22 & (37) \\
\hline Lymph nodes & 34 & $(40)$ & 21 & (53) & 30 & (50) \\
\hline Peritoneum & 23 & (27) & 12 & (30) & 19 & (32) \\
\hline Others & 11 & (13) & 11 & (28) & 10 & (I7) \\
\hline \multicolumn{7}{|l|}{ KRAS status } \\
\hline Wild & 22 & (26) & 14 & (35) & 33 & (55) \\
\hline Mutant & 11 & (13) & 13 & (33) & 18 & (30) \\
\hline Unknown & 52 & (6I) & 13 & (33) & 9 & (I5) \\
\hline
\end{tabular}

Abbreviations: BV, bevacizumab; ECOG, Eastern Cooperative Oncology Group.

statistical significances between the three arms (log-rank; $P=0.254)$. However, PFS in the XELOX arm was slightly shorter than those in the FF4 and FF6 arms. We could not estimate OS, because the median follow-up time was short and because the number of events was very few at the cut-off date. Figure 3 shows the relationship between EOTR patients and non-EOTR patients in terms of PFS. EOTR patients had a better PFS than non-EOTR patients (log-rank; $P=0.004$ ). This result means that EOTR patients with MCRC receiving oxaliplatin-based regimens plus BV had prolonged PFS.

Table 2 Treatment outcomes

\begin{tabular}{|c|c|c|c|c|c|c|}
\hline & \multicolumn{2}{|c|}{ FOLFOX4 + bevacizumab } & \multicolumn{2}{|c|}{ mFOLFOX6 + bevacizumab } & \multicolumn{2}{|c|}{ XELOX + bevacizumab } \\
\hline & $n=85$ & (\%) & $n=40$ & (\%) & $n=60$ & (\%) \\
\hline Response rate & & 61.2 & & 72.5 & & 75.0 \\
\hline$(95 \% \mathrm{Cl})$ & & $(50.6-71.8)$ & & $(58.0-87.0)$ & & $(63.7-86.3)$ \\
\hline \multicolumn{7}{|l|}{ Best overall response } \\
\hline Complete response & 6 & (7) & 3 & $(8)$ & 3 & (5) \\
\hline Partial response & 46 & (54) & 26 & $(65)$ & 42 & (70) \\
\hline Stable disease & 29 & (34) & 6 & $(15)$ & 11 & $(\mid 8)$ \\
\hline Progressive disease & 3 & (4) & 2 & (5) & 2 & (3) \\
\hline Not evaluable & 1 & (I) & 3 & (8) & 2 & (3) \\
\hline R0 resection & 9 & $(\mathrm{II})$ & 2 & $(5)$ & 7 & $(12)$ \\
\hline \multicolumn{7}{|l|}{ Treatment courses } \\
\hline Median & 21 & & 22 & & 15 & \\
\hline (Range) & $(2-98)$ & & $(I-5 I)$ & & $(1-30+)$ & \\
\hline Second-line chemotherapy & 62 & (73) & 29 & (73) & 45 & (75) \\
\hline
\end{tabular}

Abbreviation: $\mathrm{Cl}$, confidence interval. 


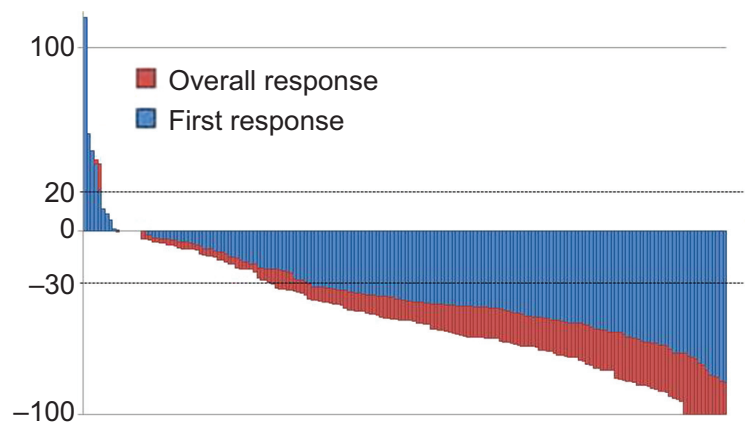

Figure I Waterfall plot analysis.

Notes: Waterfall plot of relative changes in maximum tumor diameter at the first examination (within 12 weeks, blue bars), and the best response point during chemotherapy (red bars) according to RECIST version I.0. Among 185 patients with metastatic colorectal cancer, 117 patients $(63.2 \%)$ achieved early response at the first evaluation and 126 patients $(68.1 \%$ ) achieved complete or partial response in response to oxaliplatin-based chemotherapy plus bevacizumab as first-line treatment. Abbreviation: RECIST, Response Evaluation Criteria in Solid Tumors Committee.

\section{Univariate and multivariate analyses of predictors of PFS}

Univariate and multivariate Cox proportional hazards regression was used to assess the association between factors of interest and PFS. In the univariate analysis, resection of primary site, advanced stage at diagnosis, liver metastasis, lung metastasis, and EOTR predicted PFS (Table 3). To evaluate the independent predictive effect of oxaliplatin-based regimens plus BV, a multivariate Cox regression analysis was performed. The results revealed that resection of primary site (HR, 0.394; 95\% CI: 0.209-0.742; $P=0.004$ ), liver

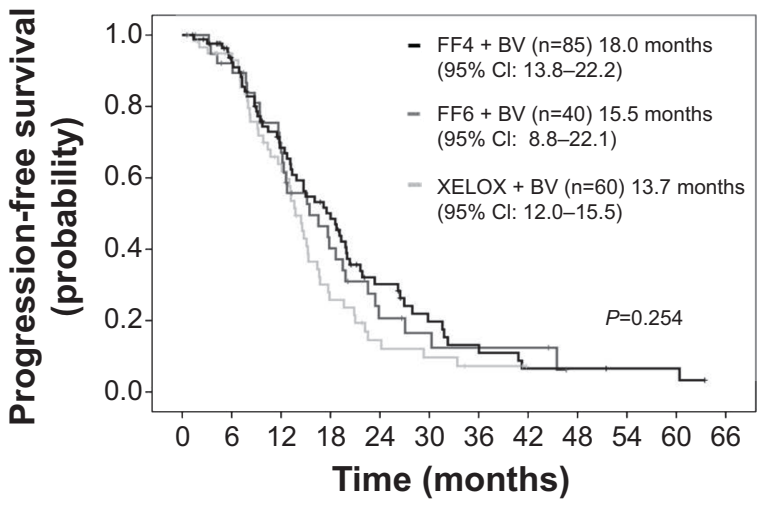

\section{Number at risk}

\begin{tabular}{lllllllllllll}
\hline $\mathrm{FF} 4+\mathrm{BV}$ & 85 & 68 & 45 & 32 & 16 & 9 & 6 & 3 & 3 & 2 & 2 & 0 \\
$\mathrm{FF6}+\mathrm{BV}$ & 40 & 34 & 24 & 13 & 6 & 4 & 3 & 3 & 0 & & & \\
XELOX + BV & 60 & 50 & 31 & 12 & 6 & 4 & 2 & 0 & & & & \\
\hline
\end{tabular}

Figure 2 Progression-free survival between three oxaliplatin-based regimens. Note: Kaplan-Meier survival curves of progression-free survival from the start of oxaliplatin-based chemotherapy plus BV as first-line chemotherapy.

Abbreviations: BV, bevacizumab; $\mathrm{Cl}$, confidence interval; FF4, FOLFOX4; FF6, mFOLFOX6.

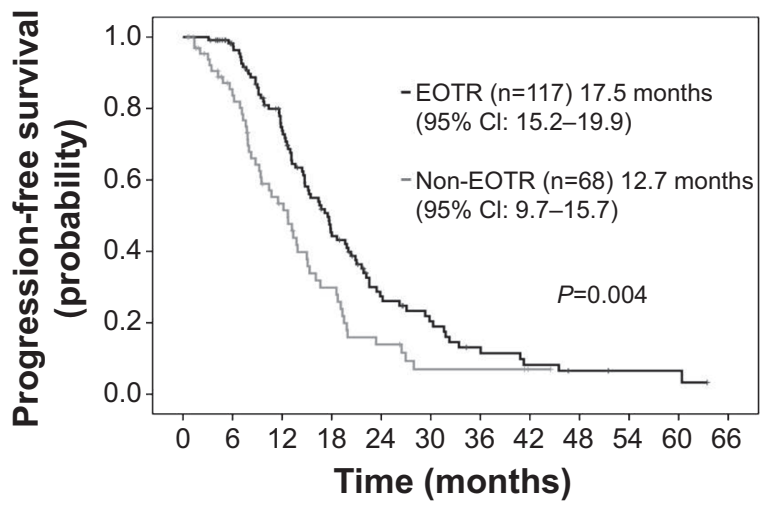

\section{Number at risk}

$\begin{array}{lllllllllllll}\text { EOTR } & 117 & 104 & 72 & 42 & 21 & 14 & 8 & 5 & 3 & 2 & 2 & 0 \\ \text { Non-EOTR } & 68 & 48 & 28 & 15 & 7 & 3 & 3 & 1 & 0 & & & \end{array}$

Figure 3 Progression-free survival in EOTR or not.

Note: Kaplan-Meier survival curves of progression-free survival in EOTR and nonEOTR patients.

Abbreviations: $\mathrm{Cl}$, confidence interval; EOTR, early objective tumor response.

metastasis (HR, 1.722; 95\% CI: 1.056-2.807; $P=0.029)$, lung metastasis (HR, $1.973 ; 95 \% \mathrm{CI}: 1.301-2.994 ; P<0.001$ ), and EOTR (HR, 0.498; 95\% CI: 0.321-0.773; $P=0.002$ ) showed independent predictive roles for PFS. On the other hand, PFS did not vary with KRAS status.

\section{Safety}

Table 4 shows the incidence of grade $3 / 4$ adverse events in each arm. The FF4 and FF6 arms were associated with a higher incidence of grade 3/4 neutropenia when compared with the XELOX arm. Grade 3 hand-foot syndrome was more frequent in the XELOX arm. With regard to BV-related adverse events, hypertension was observed at the same incidence in each arm, and the incidence of other adverse events, such as bleeding, arterial or venous thrombosis, and proteinuria, was very low. The toxicity was tolerable, and there were no treatment-related deaths within 30 days.

\section{Discussion}

This retrospective study demonstrated that there was no difference among three oxaliplatin-based regimens with BV with regard to PFS in patients with MCRC. Each regimen contributed a high ORR and excellent PFS. In addition, the toxicities of all regimens were tolerable.

The ORR in this study was $68.1 \%$ (126/185 patients), which is similar to that in a previous Phase II report in Japanese MCRC patients who received XELOX + BV (ORR, 72\%). ${ }^{18} \mathrm{R} 0$ resection rate after chemotherapy was over $10 \%$ in the FF4 and XELOX arms, while only $5 \%$ in 
Table 3 Univariate and multivariate analysis of factors related to progression-free survival

\begin{tabular}{|c|c|c|c|c|c|c|}
\hline \multirow[t]{2}{*}{ Parameter } & \multicolumn{3}{|c|}{ Univariate } & \multicolumn{3}{|c|}{ Multivariate } \\
\hline & HR & $95 \% \mathrm{Cl}$ & $P$-value & HR & $95 \% \mathrm{CI}$ & $P$-value \\
\hline Age $>60$ years & 1.022 & $0.727-1.437$ & 0.9 & & & \\
\hline Sex: male versus female & 0.975 & $0.693-|.37|$ & 0.885 & & & \\
\hline PS: 0 versus I & 1.153 & $0.468-2.836$ & 0.757 & & & \\
\hline Primary site: colon versus rectum & 0.89 & $0.6 \mathrm{II}-1.297$ & 0.544 & & & \\
\hline Resection of primary site & 0.47 & $0.293-0.754$ & 0.002 & 0.394 & $0.209-0.742$ & 0.004 \\
\hline Advanced versus recurrence & 0.659 & $0.444-0.978$ & 0.038 & 0.882 & $0.531-1.464$ & 0.627 \\
\hline Liver metastasis & 1.462 & $1.037-2.059$ & 0.003 & 1.722 & $1.056-2.807$ & 0.029 \\
\hline Lung metastasis & 2.074 & $1.464-2.939$ & $<0.001$ & 1.973 & $1.30 \mathrm{I}-2.994$ & $<0.001$ \\
\hline Lymph node metastasis & 1.132 & $0.805-1.593$ & 0.476 & & & \\
\hline Peritoneal metastasis & 0.879 & $0.615-1.255$ & 0.477 & & & \\
\hline FOLFOX arm versus $X E L O X$ arm & 0.744 & $0.518-1.068$ & 0.109 & & & \\
\hline KRAS wild-type & 0.822 & $0.53-1.274$ & 0.38 & & & \\
\hline Early objective tumor response & 0.597 & $0.419-0.85$ & 0.004 & 0.498 & $0.321-0.773$ & 0.002 \\
\hline
\end{tabular}

Notes: Early objective tumor response is defined as tumor shrinkage $\geq 30 \%$ in the sum of the longest diameters of target lesions when compared with baseline within 12 weeks. The FOLFOX arm received FOLFOX 4 or mFOLFOX6 + bevacizumab; the XELOX arm received XELOX + bevacizumab.

Abbreviations: $\mathrm{Cl}$, confidence interval; $\mathrm{HR}$, hazard ratio; PS, performance status.

the FF6 arm. We considered the reason for the difference in the $\mathrm{R} 0$ resection rate in each arm was that liver metastasis in FF6 arm (30\%) was less than in FF4 and XELOX arms (53\% and $62 \%$, respectively). Therefore, we were not able to conclude that FF6 was inferior to FF4 or XELOX therapy with regard to tumor shrinkage.

In terms of safety, the incidence of hematological and non-hematological grade $3 / 4$ adverse events in the XELOX arm was similar to that described in previous reports. ${ }^{14-16,18}$ In contrast, grade $3 / 4$ neutropenia occurred in $55 \%$ of patients in the FF4 arm and in $58 \%$ of patients in the FF6 arm, which is more common than that seen in previous studies, such as the NO16966 study (grade 3/4 neutropenia occurred in $43 \%$ in FOLFOX 4 arm) ${ }^{14}$ However, febrile neutropenia was found in only one patient in the FF4 arm. Despite the higher incidence of neutropenia in the FF4 and FF6 arms, the RDI of oxaliplatin was over $50 \%$ and that of 5 -FU was over $70 \%$. These data indicate that the hematological toxicities were manageable in each arm. Non-hematological toxicities in the FF4 and FF6 arms were similar to those seen in previous reports. ${ }^{14}$ The incidences of BV-related adverse events were consistent with those previously reported. ${ }^{22,23}$ Grade 3/4 hypertension was observed to be higher in each arm (FF4, $34 \%$; FF6, 20\%; XELOX, 27\%) than in previous reports, ${ }^{15}$ but was manageable.

PFS analysis showed no statistically significant difference between the three arms. However, the XELOX arm had a slightly shorter PFS than the FF4 and FF6 arms. This may be because more patients received surgical resection at the primary site in the FF4 and FF6 arms prior to initiation of

Table 4 Incidence of grade 3/4 adverse events according to CTCAE version 4.0

\begin{tabular}{|c|c|c|c|c|c|c|}
\hline & \multicolumn{2}{|c|}{ FOLFOX4 + bevacizumab } & \multicolumn{2}{|c|}{ mFOLFOX6 + bevacizumab } & \multicolumn{2}{|c|}{ XELOX + bevacizumab } \\
\hline & $n=85$ & $(\%)$ & $n=40$ & $(\%)$ & $n=60$ & (\%) \\
\hline Neutropenia & 47 & $(55)$ & 23 & $(58)$ & 7 & $(12)$ \\
\hline Anemia & 0 & $(0)$ & 0 & $(0)$ & 1 & $(2)$ \\
\hline Thrombocytopenia & 0 & $(0)$ & 0 & $(0)$ & 1 & $(2)$ \\
\hline Febrile neutropenia & 1 & (I) & 0 & $(0)$ & 0 & $(0)$ \\
\hline Neurosensory toxicity & 2 & $(2)$ & 4 & $(10)$ & 7 & (I2) \\
\hline Hand-foot syndrome & 0 & $(0)$ & 0 & $(0)$ & 7 & $(12)$ \\
\hline Diarrhea & 1 & (I) & 3 & $(8)$ & 6 & $(10)$ \\
\hline Nausea/vomiting & 3 & (4) & 2 & (5) & 5 & (8) \\
\hline Fatigue & 3 & (4) & 3 & (8) & 0 & (0) \\
\hline Hypertension & 29 & (34) & 8 & $(20)$ & 16 & (27) \\
\hline Bleeding & 0 & (0) & 0 & $(0)$ & 0 & (0) \\
\hline Arterial thrombosis & 0 & $(0)$ & 0 & (0) & 0 & (0) \\
\hline Venous thrombosis & 4 & (5) & 0 & (0) & 3 & (5) \\
\hline Proteinuria & 0 & $(0)$ & 0 & $(0)$ & 1 & $(2)$ \\
\hline
\end{tabular}

Abbreviation: CTCAE, Common Toxicity Criteria for Adverse Events. 
chemotherapy than in the XELOX arm. McCahill et al reported the results of a Phase II trial (NSAPB trial C-10) of patients with MCRC presenting with an asymptomatic intact primary tumor (IPT). ${ }^{24}$ The aim of the study was to determine whether surgical resection of the asymptomatic IPT was needed before initiation of FOLFOX plus BV in patients with MCRC. The rate of major morbidity, defined as any event related to the IPT necessitating surgery or leading to death, was examined as the primary endpoint. Ninety patients with MCRC and asymptomatic IPT were enrolled and received FF6 plus BV as first-line chemotherapy. The incidence of major morbidity events was $16.3 \%$, which was considered acceptable. The median OS was 19.9 months (95\% CI: 15.0-27.2 months), which was shorter than that observed in another Phase III trial of patients treated with FOLFOX plus BV (OS, 24.5 months; 95\% CI: 20.4-24.5 months). ${ }^{25}$ Therefore, we could not conclude whether surgical resection of asymptomatic IPT prior to chemotherapy was necessary based on the results from McCahill et al's study alone. According to our study, resection of the primary site might be associated with better PFS and OS, because it was extracted as an independent factor for PFS in both univariate and multivariate analyses. An ongoing prospective trial (JCOG1007) is analyzing the benefit on survival of surgical resection of primary sites in MCRC before initiation of chemotherapy and might help resolve this issue.

PFS in our study was longer than that in previous Phase III studies. This may be because we chose reintroduction of oxaliplatin. If patients suffered severe neurosensonary toxicity, we stopped oxaliplatin and continued chemotherapy consisting of BV plus 5FU/FA or capecitabine. However, when the tumors tended to regrow within stable disease and the patients recovered from neurosensory toxicity less than grade 1, we reintroduced oxaliplatin to the patients. Reintroduction of oxaliplatin is often performed in clinical practice in Japan. In fact, 95 patients stopped oxaliplatin administration because of neurosensory toxicity, of whom 53 patients $(58 \%)$ had oxaliplatin subsequently reintroduced. Moreover, we continued the first-line treatment up to baseline progressive disease when tumor growth was slow, even if progressive disease was achieved according to the RECIST criteria. These data suggest that the long-term use of oxaliplatin resulted in good PFS.

Prognostic factors are often assessed in OS. A previous study assessed a total of 3,825 MCRC patients treated with 5-FU and demonstrated three risk groups according to four prognostic factors: ECOG PS, number of tumor sites, white blood cell count, and ALP level. ${ }^{26}$ On the other hand, recent reports showed a relationship between ETS/EOTR and not only OS but also PFS. ${ }^{19-21}$ We considered that a prognostic factor was important to PFS in our study because we evaluated EOTR in patients with MCRC, so we assessed prognostic factors for PFS in MCRC patients. In multivariate analysis, we found that previous primary site resection was a predictive marker of PFS in patients treated with oxaliplatinbased chemotherapy containing BV. Most patients in the FF4 and FF6 arms underwent operation at the primary site, even though the patients had no symptoms, such as obstruction or bleeding. In contrast, the rate of primary site resection was $72 \%$ in the XELOX arm. In our institute, patients without obstruction or bleeding at the primary site were given the XELOX regimen immediately as first-line chemotherapy, because the XELOX regimen does not require central venous access to introduce chemotherapy. Patients without advanced disease at the primary site might be in better condition when receiving chemotherapy, because patients receiving primary site resection would have less tumor burden than those who were not candidates for primary site resection. In recurrence cases, the volume of disease at the metastasis site might be smaller than that in advanced cases because of early detection of recurrence. A prospective randomized trial including MCRC patients with and without primary site disease is required to examine whether primary site resection is a predictive marker of PFS.

The VEGF signaling pathway consists of five glycoproteins from the VEGF family (VEGF-A, VEGF-B, VEGF-C, VEGF-D, and placental growth factor [PIGF]), three receptors (VEGF receptor [VEGFR]-1, VEGFR-2, and VEGFR-3), and two co-receptors (neuropilin [NRP]-1 and NRP-2). ${ }^{27,28}$ VEGF-A can bind to VEGFR-1, VEGFR-2, and NRP-1. VEGF-A binding to VEGFR-2 is considered to be the key signaling pathway that mediates angiogenesis. ${ }^{29} \mathrm{BV}$ binds to VEGF-A with high affinity, and the BV-VEGF-A complex blocks the binding of VEGF-A to VEGFR, thereby inhibiting the VEGF signaling pathway. Liver and lung metastases arise via hematogenous spread. Multivariate analysis in this study indicated a relationship between better PFS and liver or lung metastases. However, we could not conclude that BV had more activity against hematogenous metastases than lymphogenous metastasis from our result.

In AVF2107 and N9741, the investigators suggested that ORR does not predict PFS and OS in response to standard chemotherapy with or without BV. ${ }^{30}$ However, several authors recently demonstrated that ETS was a good clinical predictive marker in patients with MCRC who were treated with cetuximab- or BV-containing chemotherapy. ${ }^{19,31-33}$ 
Muro et al showed that the rate of tumor shrinkage $\geq 30 \%$ in MCRC patients treated with XELOX + BV was $41.8 \%$ at 6 weeks and was $70.9 \%$ at 12 weeks after initiation of chemotherapy. ${ }^{31}$ Those investigators did not evaluate the relationship between EOTR and survival but did demonstrate good PFS and OS (PFS, 11.0 months; OS, 27.4 months). ${ }^{18}$ In addition, Ichante et al reported that ETS at 8 weeks strongly correlated with better survival in patients with MCRC who received capecitabine plus irinotecan (XELIRI) or intravenous bolus and infusional 5-FU/FA plus irinotecan (FOLFIRI) plus BV as first-line treatment, according to both univariate and multivariate analyses. ${ }^{32}$ In the PRIME trial, 656 patients with $K R A S$-WT MCRC received FOLFOX with or without panitumumab. ${ }^{33}$ Patients with EOTR had better PFS and OS than those with non-EOTR. Moreover, Sommeijer et al showed the prognostic value of EOTR for MCRC patients undergoing first-line chemotherapy. ${ }^{21}$ Those investigators reported that good PFS and OS were related to EOTR, which was evaluated at 6-8 weeks and at 12 weeks. These studies suggest that EOTR might have utility in predicting PFS and OS in patients with MCRC. We chose 12 weeks after initiation of treatment as the optimal point to assess EOTR in order to assess the effect of chemotherapy at the first evaluation conducted in routine clinical practice. In previous pivotal studies, it seemed that ORR might be lower in response to BV-containing chemotherapy when compared with cetuximab-containing chemotherapy in patients with KRAS-WT MCRC. ${ }^{14,33}$ Recently, however, the PEAK study, a randomized Phase II trial comparing FOLFOX plus BV with FOLFOX plus panitumumab; the FIRE-3 study, a randomized Phase III trial comparing FOLFIRI plus BV with FOLFIRI plus cetuximab; and the CALGB/SWOG 80405 study, a randomized Phase III trial comparing chemotherapy (mFOLFOX6 or FOLFIRI) plus BV with chemotherapy plus cetuximab, demonstrated an approximately equal ORR (approximately 60\%). ${ }^{34-36}$ The ORR of our data was close to these results. We could show the clinical value of EOTR in MCRC patients. From our results, EOTR might be related to better PFS in patients with MCRC who receive first-line BV plus oxaliplatin-based chemotherapy.

One limitation of our study was that it was a small, retrospective cohort study rather than a randomized clinical trial. The patients in our study started chemotherapy at different time points, before and after the approval of mFOLFOX6 and XELOX regimens. In addition, the median follow-up time was 35.0 months in the FF4 arm, 30.0 months in the FF6 arm, and 24.2 months in the XELOX arm, but each arm could not reach the median OS by the data cut-off. Further follow-up is necessary to confirm the benefit of oxaliplatin-based chemotherapy plus BV on survival in our study. Indeed, this study had less power to assess the clinical benefit for PFS and OS when compared with a large-sample-size Phase III study, such as NO16966. ${ }^{14,15}$ Therefore, a randomized clinical trial is needed to confirm the safety and efficacy of these three oxaliplatin-based regimens plus $\mathrm{BV}$ in patients with MCRC.

\section{Conclusion}

This is the first study to compare the three oxaliplatin-based regimens plus BV. We showed similar efficacy and feasibility between the FF4, FF6, and XELOX arms. Moreover, EOTR was useful for estimating the benefit of oxaliplatinbased regimens plus BV as first-line treatment in patients with MCRC. In the future, we might discuss whether or not to change the treatment regimen according to EOTR or non-EOTR. We will need a prospective study in order to determine the utility of EOTR in PFS and OS in patients with MCRC treated with BV plus oxaliplatin-based regimens.

\section{Acknowledgments}

We thank the staff of the Ambulatory Treatment Center at the Cancer Institute Hospital of the Japanese Foundation for Cancer Research for their management of the patients. The results of this study were presented at the 2012 Gastrointestinal Cancers Symposium (abstract number: 687).

\section{Disclosure}

The authors report no conflicts of interest in this work.

\section{References}

1. Globocan. Colorectal cancer fact sheet 2008. Available from: http://globocan.jarc.fr/factsheets/cancers/colorectal.asp. Accessed May 1, 2014.

2. Schmoll HJ, Büchele T, Grothey A, Dempke W. Where do we stand with 5-fluorouracil? Semin Oncol. 1999;26:589-605.

3. de Gramont A, Figer A, Seymour M, et al. Leucovorin and fluorouracil with or without oxaliplatin as first-line treatment in advanced colorectal cancer. J Clin Oncol. 2000;18:2938-2947.

4. Goldberg RM, Sargent DJ, Morton RF, et al. A randomized controlled trial of fluorouracil plus leucovorin, irinotecan, and oxaliplatin combinations in patients with previously untreated metastatic colorectal cancer. J Clin Oncol. 2004;22:23-30.

5. Miwa M, Ura M, Nishida M, et al. Design of a novel oral fluoropyrimidine carbamate, capecitabine, which generates 5-fluorouracil selectively in tumours by enzymes concentrated in human liver and cancer tissue. Eur J Cancer. 1998;34:1274-1281.

6. Schüller J, Cassidy J, Dumont E, et al. Preferential activation of capecitabine in tumor following oral administration to colorectal cancer patients. Cancer Chemother Pharmacol. 2000;45:291-297.

7. Van Cutsem E, Twelves C, Cassidy J, et al; Xeloda Colorectal Cancer Study Group. Oral capecitabine compared with intravenous fluorouracil plus leucovorin in patients with metastatic colorectal cancer: results of a large phase III study. J Clin Oncol. 2001;19:4097-4106.

8. Van Cutsem E, Hoff PM, Harper P, et al. Oral capecitabine vs intravenous 5-fluorouracil and leucovorin: integrated efficacy data and novel analyses from two large, randomised, phase III trials. Br J Cancer. 2004;90:1190-1197. 
9. Hoff PM, Ansari R, Batist G, et al. Comparison of oral capecitabine versus intravenous fluorouracil plus leucovorin as first-line treatment in 605 patients with metastatic colorectal cancer: results of a randomized phase III study. J Clin Oncol. 2001;19:2282-2292.

10. Twelves C, Wong A, Nowacki MP, et al. Capecitabine as adjuvant treatment for stage III colon cancer. N Engl J Med. 2005;352:2696-2704.

11. Cassidy J, Tabernero J, Twelves C, et al. XELOX (capecitabine plus oxaliplatin): active first-line therapy for patients with metastatic colorectal cancer. J Clin Oncol. 2004;22:2084-2091.

12. Makatsoris T, Kalofonos HP, Aravantinos G, et al; Hellenic Cooperative Oncology Group. A phase II study of capecitabine plus oxaliplatin (XELOX): a new first-line option in metastatic colorectal cancer. Int $J$ Gastrointest Cancer. 2005;35:103-109.

13. Scheithauer W, Kornek GV, Raderer M, et al. Randomized multicenter phase II trial of two different schedules of capecitabine plus oxaliplatin as first-line treatment in advanced colorectal cancer. J Clin Oncol. 2003;21:1307-1312.

14. Cassidy J, Clarke S, Díaz-Rubio E, et al. Randomized phase III study of capecitabine plus oxaliplatin compared with fluorouracil/folinic acid plus oxaliplatin as first-line therapy for metastatic colorectal cancer. J Clin Oncol. 2008;26:2006-2012.

15. Saltz LB, Clarke S, Díaz-Rubio E, et al. Bevacizumab in combination with oxaliplatin-based chemotherapy as first-line therapy in metastatic colorectal cancer: a randomized phase III study. J Clin Oncol. 2008;26:2013-2019.

16. Cassidy J, Clarke S, Díaz-Rubio E, et al. XELOX vs FOLFOX-4 as first-line therapy for metastatic colorectal cancer: NO16966 updated results. Br J Cancer. 2011;105:58-64.

17. Suenaga M, Mizunuma N, Matsusaka S, Shinozaki E, Ueno M, Yamaguchi T. Retrospective analysis on the efficacy of bevacizumab with FOLFOX as a first-line treatment in Japanese patients with metastatic colorectal cancer. Asia Pac J Clin Oncol. 2014;10:322-329.

18. Doi T, Boku N, Kato K, et al. Phase I/II study of capecitabine plus oxaliplatin (XELOX) plus bevacizumab as first-line therapy in Japanese patients with metastatic colorectal cancer. Jpn J Clin Oncol. 2010;40:913-920.

19. Modest DP, Laubender RP, Stintzing S, et al. Early tumor shrinkage in patients with metastatic colorectal cancer receiving first-line treatment with cetuximab combined with either CAPIRI or CAPOX: an analysis of the German AIO KRK 0104 trial. Acta Oncol. 2013;52: 956-962.

20. Giessen C, Laubender RP, Fischer von Weikersthal L, et al. Early tumor shrinkage in metastatic colorectal cancer: retrospective analysis from an irinotecan-based randomized first-line trial. Cancer Sci. 2013;104:718-724.

21. Sommeijer DW, Shi Q, Meyers JP, et al. Prognostic value of early objective tumor response (EOTR) to first-line systemic therapy in metastatic colorectal cancer (mCRC): individual patient data (IPD) meta-analysis of randomized trials from the ARCAD database. J Clin Oncol. 2013;31(suppl; abstr 3520).

22. Grothey A, Sugrue MM, Purdie DM, et al. Bevacizumab beyond first progression is associated with prolonged overall survival in metastatic colorectal cancer: results from a large observational cohort study (BRiTE). J Clin Oncol. 2008;26:5326-5334.
23. Van Cutsem E, Rivera F, Berry S, et al; First BEAT investigators Safety and efficacy of first-line bevacizumab with FOLFOX, XELOX, FOLFIRI and fluoropyrimidines in metastatic colorectal cancer: the BEAT study. Ann Oncol. 2009;20:1842-1847.

24. McCahill LE, Yothers G, Sharif S, et al. Primary mFOLFOX6 plus bevacizumab without resection of the primary tumor for patients presenting with surgically unresectable metastatic colon cancer and an intact asymptomatic colon cancer: definitive analysis of NSABP trial C-10. J Clin Oncol. 2012;30:3223-3228.

25. Hecht JR, Mitchell E, Chidiac T, et al. A randomized phase IIIB trial of chemotherapy, bevacizumab, and panitumumab compared with chemotherapy and bevacizumab alone for metastatic colorectal cancer. $J$ Clin Oncol. 2009;27:672-680.

26. Köhne CH, Cunningham D, Di Costanzo F, et al. Clinical determinants of survival in patients with 5-fluorouracil-based treatment for metastatic colorectal cancer: results of a multivariate analysis of 3,825 patients. Ann Oncol. 2002;13:308-317.

27. Ellis LM, Hicklin DJ. VEGF-targeted therapy: mechanisms of antitumour activity. Nat Rev Cancer. 2008;8:579-591.

28. Sun W. Angiogenesis in metastatic colorectal cancer and the benefits of targeted therapy. J Hematol Oncol. 2012;5:63.

29. Kerbel RS. Tumor angiogenesis. N Engl J Med. 2008;358: 2039-2049.

30. Grothey A, Hedrick EE, Mass RD, et al. Response-independent survival benefit in metastatic colorectal cancer: a comparative analysis of N9741 and AVF2107. J Clin Oncol. 2008;26:183-189.

31. Muro K, Doi T, Boku N, et al. Final results of bevacizumab (BV) plus capecitabine plus oxaliplatin (XELOX) as first-line therapy in Japanese patients (pts) with metastatic colorectal cancer (MCRC). ASCO-GI. 2010; abstr 466.

32. Ichante JL, Adenis A, Malka D, et al. Impact of early tumor shrinkage on long-term outcome in metastatic colorectal cancer (mCRC) treated with 5-FU + irinotecan + leucovorin (FOLFIRI) or capecitabine + irinotecan (XELIRI) plus bevacizumab. ESMO. 2011; abstr 6094

33. Douillard JY, Siena S, Tabernero J, et al. Understanding the value of response and early tumor shrinkage in patients with wild-type KRAS metastatic colorectal cancer treated with panitumumab plus FOLFOX. Ann Oncol. 2012;23:suppl 9; abstr 558p.

34. Schwartzberg LS, Rivera F, Karthaus M, et al. PEAK: a randomized, multicenter phase II study of panitumumab plus modified fluorouracil, leucovorin, and oxaliplatin (mFOLFOX6) or bevacizumab plus mFOLFOX6 in patients with previously untreated, unresectable, wild-type KRAS exon 2 metastatic colorectal cancer. J Clin Oncol. 2014;32:2240-2247.

35. Heinemann V, von Weikersthal LF, Decker T, et al. FOLFIRI plus cetuximab versus FOLFIRI plus bevacizumab as first-line treatment for patients with metastatic colorectal cancer (FIRE-3): a randomised, open-label, phase 3 trial. Lancet Oncol. 2014;15:1065-1075.

36. Venook AP, Niedzwiecki D, Lenz HJ, et al. CALGB/SWOG 80405: Phase III trial of irinotecan/5-FU/leucovorin (FOLFIRI) or oxaliplatin/5-FU/leucovorin (mFOLFOX6) with bevacizumab or cetuximab for patients with KRAS wild-type untreated metastatic adenocarcinoma of the colon or rectum (MCRC). J Clin Oncol. 2014;32:suppl, abstr LBA3.
OncoTargets and Therapy

\section{Publish your work in this journal}

OncoTargets and Therapy is an international, peer-reviewed, open access journal focusing on the pathological basis of all cancers, potential targets for therapy and treatment protocols employed to improve the management of cancer patients. The journal also focuses on the impact of management programs and new therapeutic agents and protocols on

\section{Dovepress}

patient perspectives such as quality of life, adherence and satisfaction. The manuscript management system is completely online and includes a very quick and fair peer-review system, which is all easy to use. Visit http://www.dovepress.com/testimonials.php to read real quotes from published authors. 